Niki Baxter $\cdot$ Sean Duffy

\title{
The effects of a 3-month depot preparation of leuprorelin acetate on pain symptoms, bone mineral density and hormone levels in women with endometriosis_-preliminary data analysis
}

Published online: 17 April 2004

(C) Springer-Verlag Berlin / Heidelberg 2004

\begin{abstract}
The purpose of this study was to determine the effectiveness of Prostap $3 \mathrm{M}$, a 3-month depot preparation of a gonadotrophin-releasing hormone agonist, in the relief of pelvic pain symptoms caused by endometriosis and to assess its effects on bone mineral density. A prospective cohort study was carried out at St. James's University Hospital, Leeds, and included 13 women with laparoscopically diagnosed endometriosis $(n=13)$. Baseline investigations were performed to measure pain symptoms, bone mineral density and oestradiol levels. The women were then treated with 7 months of Prostap $3 \mathrm{M}$. At the end of treatment, the investigations were repeated. After the 7-month treatment phase, there was a statistically significant reduction in all the pain subset scores. The continuous GnRHa therapy resulted in a fall of serum oestradiol levels into the post-menopausal range. After treatment with Prostap $3 \mathrm{M}$, women with endometriosis showed a mean decrease of $5.57 \%$ in BMD of the lumbar spine and of $3.47 \%$ in femoral BMD. Both these falls are statistically significant. Prostap $3 \mathrm{M}$ is effective in relieving the painful symptoms associated with endometriosis, and the continuous therapy over 7 months does indeed induce a hypo-oestrogenic state. However, the effects of Prostap $3 \mathrm{M}$ on bone mineral density appear to be more detrimental than seen with monthly preparations.
\end{abstract}

Keywords Endometriosis · Pelvic pain - Gonadotrophinreleasing hormone agonists

\footnotetext{
N. Baxter

Department of Obstetrics and Gynaecology, Jessop Wing,

Tree Root Walk, Sheffield, S10 2SF, UK

S. Duffy

Department of Obstetrics and Gynaecology,

St James's University Hospital,

Beckett Street, Leeds, LS9 7TF, UK

12 Alexandra Gardens, Nether Edge, Sheffield, S11 9DQ, UK

N. Baxter (-

e-mail: nikibaxter@btinternet.co
}

\section{Introduction}

Chronic pelvic pain (CPP) is defined as pain that has been present for 6 months or longer and is a common cause of referral to gynaecology clinics. The most common cause found for the pain is endometriosis, which is characterised by ectopic endometrial tissue lying within the pelvis. There are many theories about the cause of endometriosis, including Sampson's theory of "retrograde menstruation". Diagnostic laparoscopy is the 'gold standard' investigation for endometriosis and the only way to make a definitive diagnosis of the condition. Laparoscopy may reveal the presence of endometrial deposits in the form of black spots, red polyps, white scarring, adhesions, endometriomas, etc.

Endometriosis may be treated medically or surgically. Hormone treatment of endometriosis is based on the theory that endometriotic lesions require oestradiol to continue growing as oestrogen receptors are present in endometriotic deposits. Therefore, by inducing a hypooestrogenic state, regression of the disease should occur. Gonadotrophin-releasing hormone agonists (GnRHa) were discovered in the early 1970s and first used clinically in the 1980s. Since then they have become one of the mainstay treatments for endometriosis. They act at a pituitary level, causing a fall in serum FSH and LH levels that in turn leads to a decrease in ovarian oestrogen production. GnRHa have been proved to be effective in the management of the painful symptoms associated with endometriosis [1].

Leuprorelin acetate is a widely used GnRHa in the management of endometriosis pain. At present, in the UK, it is licensed to be used in gynaecology in its monthly form (3.75 mg, Prostap SR, Wyeth). Several trials have shown a 6-month treatment of Prostap SR (six doses) to be highly effective for endometriosis-related pain. Wheeler et al. [2] reported that $55 \%$ of women with endometriosis $(n=128)$ reported complete resolution of pain after Prostap SR treatment over 24 weeks. However, at the end of 6 months of therapy with a continuous GnRHa, reductions of 2.9 to $5.6 \%$ in the bone mineral density of the lumbar spine have been reported $[3,4,5]$. 
Prostap $3 \mathrm{M}$ is a new 3-monthly depot preparation of leuprorelin acetate, $11.25 \mathrm{mg}$. It is administered in a similar way to Prostap SR, either intramuscularly or subcutaneously. The advantages of this preparation over Prostap SR are that during a 6-month treatment period, only two injections would be needed, resulting in fewer visits to hospital for the patient and therefore cutting down on clinic waiting times. Our preliminary studies have shown Prostap 3 M to be as effective as Prostap SR in reducing painful symptoms over a 6-month therapeutic phase. However, its possible adverse effects are as yet unknown. This preliminary trial investigates the effectiveness with regard to pain and the bone demineralisation effects of Prostap $3 \mathrm{M}$ and considers the results in light of available data on Prostap SR.

\section{Materials and methods}

Thirteen women were recruited into this trial. All had initially presented to the gynaecology out-patient clinic at St James's University Hospital, Leeds, Yorkshire, complaining of chronic pelvic pain for at least 6 months. Each underwent a diagnostic laparoscopy under a general anaesthetic and all were found to have mild endometriosis. After being fully informed about the trial and obtaining written consent, each woman had a Dual Energy X-ray Absorptiometry (DEXA) scan to measure baseline bone mineral density in the lumbar spine vertebrae $2-4$ and neck of the femur. A venous blood sample was taken to measure serum oestradiol levels. All of them then completed a simple $0-3$ baseline questionnaire, whereby they scored their symptoms of dysmenorrhoea, dyspareunia and pelvic pain (where $0=$ no pain and $3=$ severe pain). The women were then treated with a 7-month course of Prostap (a 1-month dose of $3.75 \mathrm{mg}$ leuprorelin acetate to establish tolerance, followed by two 3-month injections of $11.25 \mathrm{mg}$ ). At the end of the treatment phase, the pain questionnaire, DEXA scan and blood test were repeated. Cross-calibration of the DEXA equipment was performed to ensure quality control. All the results were entered into a database and statistically analysed. Significance was assumed for $P$ values of less than 0.05 .

\section{Results}

Complete data have been obtained on 13 white women. The ages ranged form 22 to 46 years with a mean of 32.7 years. All the women had grade I or II endometriosis on laparoscopy, using the American Fertility Society classification. The mean spine BMD at the start of the study was $1.261 \mathrm{~g} / \mathrm{cm}^{2}$ and the mean femur BMD was $1.043 \mathrm{~g} / \mathrm{cm}^{2}$. Baseline mean pain scores were as follows: dysmenorrhoea $2.31 \pm 1.11$, dyspareunia $1.85 \pm 0.90$ and non-cyclical pelvic pain $2.08 \pm 0.76$. All women had serum oestradiol levels within the normal pre-menopausal range.

When the group was subdivided into endometriosis grades I or II, using the revised American Fertility Society classification, there was no significant difference between the two subsets with regard to the baseline characteristics of body mass index (BMI), age, spinal or femoral BMD, or serum oestradiol levels. Grade of endometriosis also had no significant influence on baseline pain scores.

After the 7-month treatment phase, there was a statistically significant reduction in all the pain subset scores

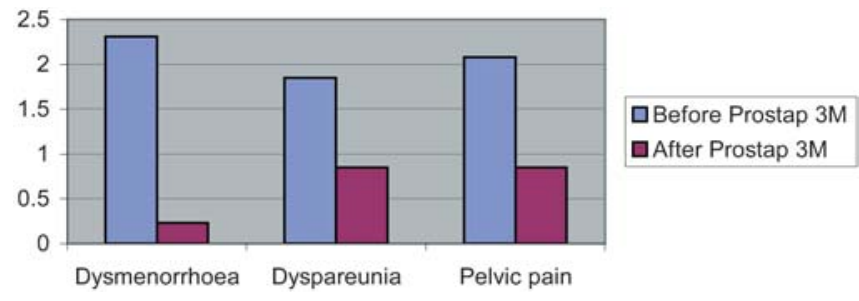

Fig. 1 Changes in mean pain scores after treatment with Prostap $3 \mathrm{M}$

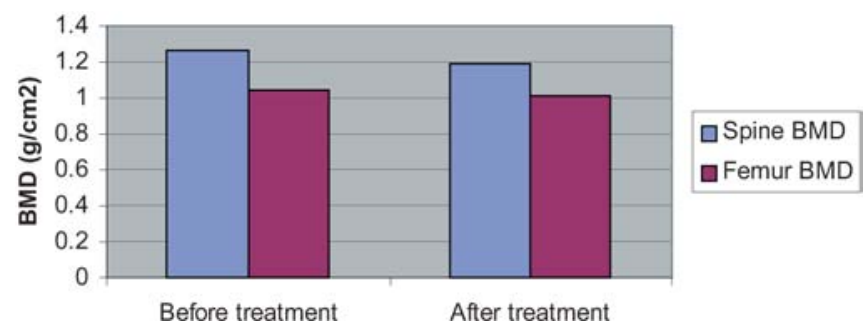

Fig. 2 Changes in BMD after Prostap 3M

(Fig. 1). There was a mean decrease in score of 2.08 for dysmenorrhoea, 1.0 for dyspareunia and 1.23 for nonmenstrual pain (Fig. 1).

The mean spine BMD post-treatment was $1.19 \pm 0.15$. This was an overall $5.57 \%$ decrease. The mean neck of femur BMD dropped to $1.04 \pm 0.15$, indicating a percentage loss of $3.47 \%$. A paired $t$-test showed that the decreases in bone mineral density in both the spine and the neck of the femur after treatment with Prostap $3 \mathrm{M}$ were statistically significant (Fig. 2).

The decrease in serum oestradiol levels was also statistically significant after treatment. At the start of the study, the mean serum oestradiol value was $226.5 \mathrm{pmol} / \mathrm{l}$. After 7 months of Prostap $3 \mathrm{M}$, the mean fell to $94.3 \mathrm{pmol} /$ 1 , which is in the post-menopausal range. The grade of endometriosis did not have any statistically significant influence on the changes in pain score, BMD or oestradiol levels.

\section{Discussion}

It has been suggested that endometriosis is associated with reduced bone mass in the periphery [6], possibly secondary to immune dysfunction. Other studies have shown that women with endometriosis have bone mineral

Table 1 Differences in mean bone mineral density $\left(\mathrm{g} / \mathrm{cm}^{2}\right)$ of the neck of femur between white women with and without endometriosis

\begin{tabular}{lll}
\hline & $\begin{array}{l}\text { No endometriosis } \\
\text { (Looker et al. [11]) }\end{array}$ & $\begin{array}{l}\text { Endometriosis } \\
\text { (Our data) }\end{array}$ \\
\hline 20-29 years & 0.858 & 1.145 \\
30-39 years & 0.825 & 1.002 \\
40-49 years & 0.791 & 0.954 \\
\hline
\end{tabular}


Table 2 A comparison of Prostap SR and Prostap $3 \mathrm{M}$ with regard to reduction in pain as a percentage

\begin{tabular}{lll}
\hline & (Leuprorelin acetate, 3.75 mg [12]) & $\begin{array}{l}\text { (Leuprorelin acetate, 11.25 mg) } \\
\text { Prostap 3M }\end{array}$ \\
\hline Prostap SR & \\
\hline Dysmenorrhoea & $98.2 \%$ & $88.5 \%$ \\
Dyspareunia & $70.5 \%$ & $73.1 \%$ \\
Non-menstrual pain & $70.2 \%$ & $55.1 \%$ \\
\hline
\end{tabular}

density levels within the range normal for age $[7,8,9$, 10].

Looker et al. [11] produced normal ranges of bone mineral density of the neck of femur based on their data from normal US adults. They took a nationally representative sample and produced tables divided into groups by sex, ethnicity and age. We compared our baseline measurement results of the mean BMD of the neck of femur in white women with endometriosis to their figures on normal healthy white women (Table 1).

In a similar way to normal healthy women, women with endometriosis showed a decreasing femoral BMD with age. However, we found that endometriosis sufferers appear to have an increased mean femoral BMD compared with normal values, and these differences are statistically different $(P=0.02)$. The grade of endometriosis does not significantly influence the BMD.

Our preliminary data shows that Prostap $3 \mathrm{M}$ is an effective treatment for the pain caused by endometriosis. The Gestrinone Italian Study Group [12] looked at the effectiveness of the monthly preparation of leuprorelin acetate, Prostap SR, in similar patients and, comparing our results with their data, Prostap $3 \mathrm{M}$ appears to be as effective as Prostap SR in terms of symptom relief $(P>0.05$; Table 2$)$.

As expected, the continuous GnRHa therapy resulted in a hypo-oestrogenic state, and this was reflected in the fall of serum oestradiol levels into the post-menopausal range.

After treatment with Prostap $3 \mathrm{M}$, women with endometriosis showed a mean decrease of $5.57 \%$ in BMD of the lumbar spine and of $3.47 \%$ in femoral BMD. Both these falls are statistically significant. Dlugi et al. [13] reported a mean loss of $3.6 \%$ in spinal bone mineral density in 15 women with endometriosis who had been treated with Prostap SR for 6 months. Hornstein et al. [14] also treated their endometriosis patients with Prostap SR and found a BMD fall of $3.2 \pm 1.8 \%$ in the lumbar spine at 6 months. Our results show a mean lumbar spine BMD decrease of $5.57 \%$, which suggests that the use of Prostap $3 \mathrm{M}$ is more detrimental than Prostap SR in terms of bone demineralisation.

\section{Conclusion}

Prostap $3 \mathrm{M}$ appears to be as effective as Prostap SR in relieving the painful symptoms associated with endometriosis. As expected, the continuous therapy over 7 months induced a hypo-oestrogenic state. The effects of Prostap $3 \mathrm{M}$ on bone mineral density seem to be more detrimental than Prostap SR. A randomised controlled trial to assess the benefits of concurrent hormone replacement therapy with Prostap $3 \mathrm{M}$ is now in progress.

\section{References}

1. Henzl MR (1988) Gonadotrophin-releasing hormone (GnRH) agonists in the management of endometriosis: a review. Clin Obstet Gynecol 31:840-856

2. Wheeler JM, Knittle JD, Miller JD (1992) Depot leuprolide versus danazol in treatment of women with symptomatic endometriosis. I. Efficacy results. Am J Obstet Gynecol 167: $1367-1371$

3. Scialli AR, Jestila KJ, Simon JA (1993) Leuprolide acetate and bone mineral density measured by quantitative digitized radiography. Fertil Steril 59:674-676

4. Surrey ES, Judd HL (1992) Reduction of vasomotor symptoms and bone mineral density loss with combined norethindrone and long-acting gonadotrophin-releasing hormone agonist therapy of symptomatic endometriosis: a prospective randomised trial. J Clin Endocrinol Metab 72:558-563

5. Finkelstein JS, Klibanski A, Schaeffer EH, Hornstein MD, Schiff I, Neer RM (1994) Parathyroid hormone for the prevention of bone loss induced by estrogen deficiency. N Eng J Med 331:1618-1623

6. Comite F, Delman M, Hutchinson-Williams K, DeCherney AH, Jensen P (1989) Reduced bone mass in reproductive-aged women with endometriosis. J Clin Endocrinol Metab 69:837842

7. Ulrich U, Murano R, Skinner M, Yin H, Chestnut CH III (1998) Women of reproductive age with endometriosis are not osteopenic. Fertil Steril 69:821-825

8. Lane N, Baptista J, Snow-Harter C (1991) Bone mineral density of the lumbar spine in endometriosis subjects compared to an age-similar control population. J Clin Endocrinol Metab 72:510-514

9. Lane N, Baptista J, Orwoll E (1991) Bone mineral density of the lumbar spine in women with endometriosis. Fertil Steril 55:537-542

10. Dochi T, Lees B, Sidhu M, Stevenson JC (1994) Bone density and endometriosis. Fertil Steril 61:175-177

11. Looker AC, Wahner HW, Dunn WL, Calvo MS, Harris TB, Heyse SP, Johnston CC, Lindsay R (1998) Updated data on proximal femur bone mineral levels of US adults. Osteoporos Int 8:468-489

12. The Gestrinone Italian Study Group (1996) Gestrinone versus a gonadotropin-releasing hormone agonist for the treatment of pelvic pain associated with endometriosis: a multicenter, randomized, double-blind study. Fertil Steril 66:911-919

13. Dlugi AM, Miller JD, Knittle J, Lupron Study Group (1990) Lupron depot (leuprolide acetate for depot suspension) in the treatment of endometriosis: a randomised, placebo-controlled, double-blind study. Fertil Steril 54:419-427

14. Hornstein MD, Surrey ES, Weisberg GW, Casino LA (1998) Leuprolide acetate depot and hormonal add-back in endometriosis: a 12-month study. Lupron Add-Back Study Group. Obstet Gynecol 91:16-24

15. Blake GM, Fogelman I (2003) The reporting of DXA scans. Osteoporos Rev 11:4-6 